\title{
A new method of autologous fascia lata replacement for aortic valvular disease
}

\author{
ERICHAUF and CHARLES-HENRI CHALANT \\ Department of Cardiovascular Surgery, University of Louvain Medical School, Louvain, Belgium
}

A new technique is described using homologous fascia lata for the construction of an aortic valve without prosthetic support and resulting in the simple and rapid production of the valve. The latter is nearing normal anatomical and physiological conditions. The successful use of such a valve in a case of acute bacterial endocarditis is described.

Based on Senning's (1967) technique of aortic valve reconstruction with fascia lata, and on our own experience with Edwards' (Edwards, Karp, Robillard, and Kerr, 1969) and Ionescu's methods of fashioning fascia lata valves (Ionescu, Ross, Deac, Grimshaw, Taylor, Whitaker, and Wooler, 1970) we have developed a new procedure for the construction of aortic fascia lata valves without prosthetic support.

Such a valve has the advantage of imitating the normal anatomy and physiology of the aortic valve as closely as possible. With the valves fixed on rigid rings we have observed pressure gradients of up to $50 \mathrm{mmHg}$. Our method of valvular replacement was used even in a patient with active endocarditis, thus avoiding the introduction of foreign material.

Two conditions are required for a correct aortic valvular replacement without prosthetic support: (1) the measurements of the new valve must correspond to those of the aortic annulus of the receiver. As there is no way of measuring these before opening the aorta, the technique has to be expeditious ;

(2) the technique of insertion of the valve must be simple.

On the basis of these requirements we have developed a method that permits the rapid construction (15 minutes) and easy insertion of a 'tailored' aortic valve made of fascia lata.

\section{DESCRIPTION OF MATERIAL ${ }^{1}$}

The material used consists of a series of paired metallic plates and a metallic rod. In a given pair the plates are the mirror-image of each other (Fig. 1). They are numbered from 6 to 14. These numbers corre-

1 Available from Dr. E. Hauf, Hôpital Cantonel, Geneva, Switzerland. spond to Starr's aortic obturators. Each pair of plates has a smooth external surface. The internal surfaces contain a longitudinal groove and a slit or cut-out, representing the pattern of an aortic valve, transected $\vec{\vartheta}$ longitudinally at one commissure and flattened out.

This contour will be called 'festoon' in the text. The groove is situated $2 \mathrm{~mm}$ above the three convexities or bottoms of the cusps. A system of spikes and corresponding holes allows for stable maintenance of both plates against each other. The width of the $ᄋ$ plates varies with each number. A strip of fascia $\varrho$ patterned on a given plate will have the required $\overrightarrow{\overrightarrow{0}}$ measurements.

The slit or cut-out (festoon) in each plate has been calculated so as to produce ultimately a continent aortic valve. These calculations are based on the work of Reid (1970) concerning the anatomy of the sinus of Valsalva.

\section{TECHNIQUE OF VALVE CONSTRUCTION}

A strip of fascia lata $(15$ by $7 \mathrm{~cm})$ is taken at the beginning of the operation from the lateral aspect of
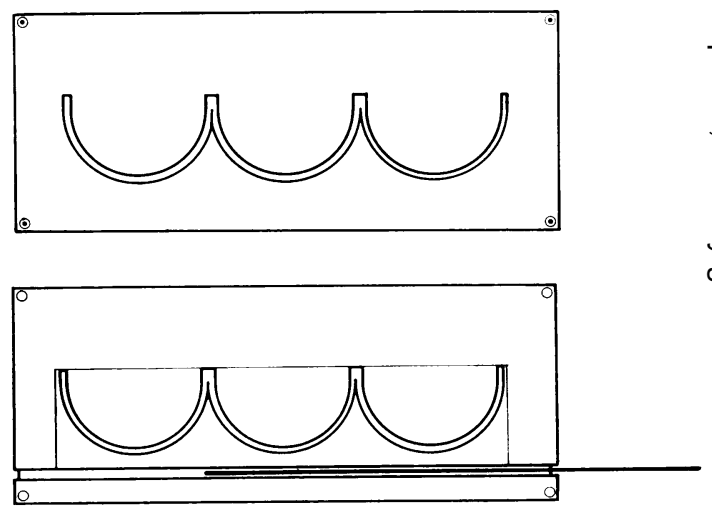

FIG. 1. Plates and rod described in the text. 
the thigh. This strip is carefully trimmed of all fatty and loose connective tissue. Attention is paid not to disturb the interlacing morphology of the fascia. The superficial adventitial layer through which the capillaries run is equally respected. A border rigorously parallel to the longitudinal fibres of the fascia is then cut with a scalpel. Both ends of the strip are sectioned perpendicular to the fibres in order to obtain a rectangle of 12 by $6 \mathrm{~cm}$ with three sharply delineated borders.

The strip is then stored in a cup containing neomycin in saline solution. Following aortotomy and excision of the diseased valve the valvular ring is calibrated using Starr's obturators. Each obturator size corresponds to two metallic plates, as previously described. The excess of fascia is excised, using a chosen plate as a convenient guide by placing it on top of the fascial rectangle. Following this we place one half of the precisely fashioned rectangle on the internal surface of one of the two identical plates, so that one of the longitudinal borders of the fascia corresponds exactly to a line passing along the top of three festoons.

A cylindrical rod is then placed across the fascia longitudinally (Fig. 2) at the level of the groove, and the remaining half of the fascial rectangle is then folded over using the rod as a hinge. The fascial rectangle is thus divided into two exactly superimposed, connected (around the rod) halves (Fig. 3).

The second identical plate is then placed on top of the folded fascia, thus immobilizing it-like a sandwich-between the two consolidated plates.

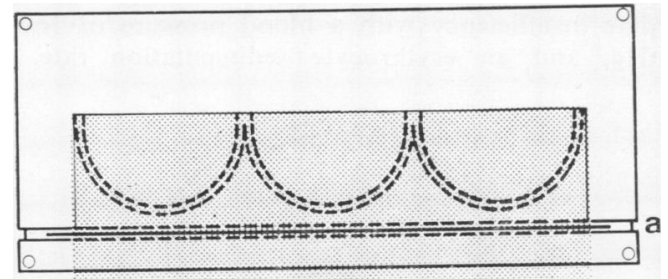

FIG. 2. The strip of fascia is positioned on the plate and the rod is introduced at $(a)$.

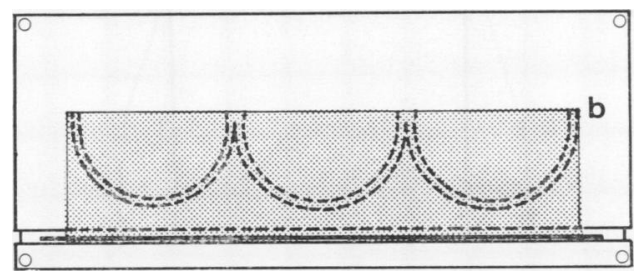

FIo. 3. The remaining half of fascia is folded around the rod. The free borders of the fascial strip are exactly superimposed at (b).
The two layers of fascia are then sutured together first by four individual $U$-stitches of $3 / 0$ mersilene introduced through the cut-out in the plates, at the apex of each festoon (commissure). One needle is threaded in a sewing machine fashion through the slit or cut-out in the plates, thus consolidating the two sheets of fascia as far as B (Fig. 4). After tying the sutures, the same procedure is repeated in reverse

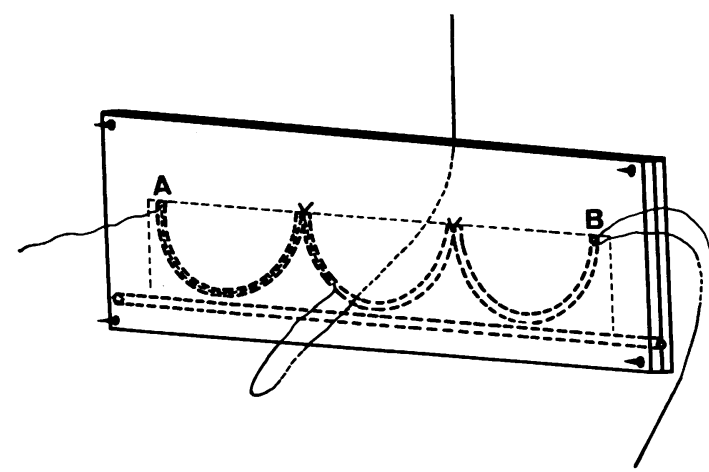

FIG. 4. The superimposed fascial layers are sutured together, following the cut-out of the plates.

with another atraumatic needle. The two plates are then separated and the metallic rod is taken out. The excess tissue at each end is trimmed and the two superimposed lateral extremities of the rectangle are sutured to each other by a double continuous suture. This suture line transforms the longitudinal superimposed fascial sheets into a cylinder. A pinching suture at the crotch of two joining commissures is fashioned by a U-stitch so that the free borders are levelled. Finally, the external sheet of fascia is excised along the free border of the sewing machine suture lines, leaving a double crown of tissue at the base of the valve (Fig. $5 \mathrm{a}, \mathrm{b}, \mathrm{c}$ ). The valve is thus ready, some 15 minutes after calibrating the receiver's ring.

\section{INSERTION OF THE VALVE}

The valve, turned outside in, is introduced through the aorta into the left ventricle so that its inferior edge, represented by the double ring or crown of fascia, is situated a few millimetres below the anatomical insertion line of the aortic valves, and the commissures of the new valve are in the prolongation of the longitudinal axes passing through the natural commissures. We then suture the ring of fascia to the cardiac tissue below the aortic ring by three continuous $3 / 0$ sutures interrupted at the level of each commissure (Fig. 6).

The valve is then returned into the aorta and the free border of external fascia along the sewing machine suture is sutured to the aortic ring by a $3 / O$ stitch, starting at the bottom of each pigeon nest (cusp) and running along the insertion line up to the 


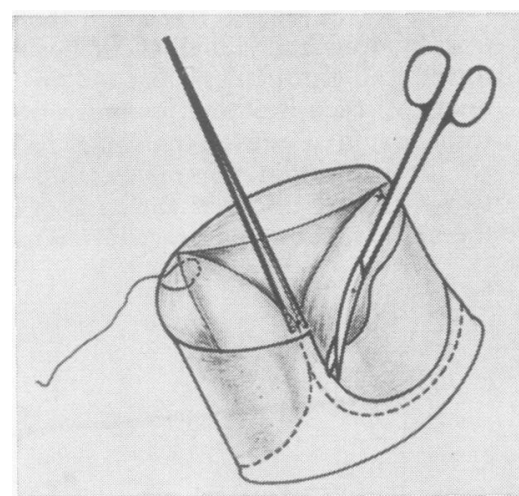

(a)

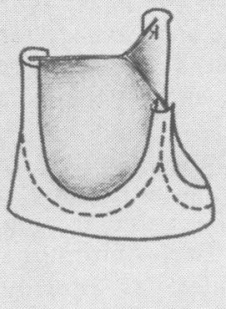

(b)

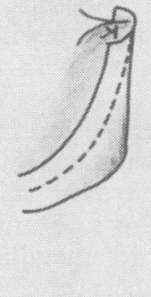

(c)

FIG. 5. (a) The external layer of fascia is excised along the suture line leaving a double crown of fascia at the bottom. The commissures are levelled with a U-stitch. (b) The finished product. (c) Detail of the commissure suture.

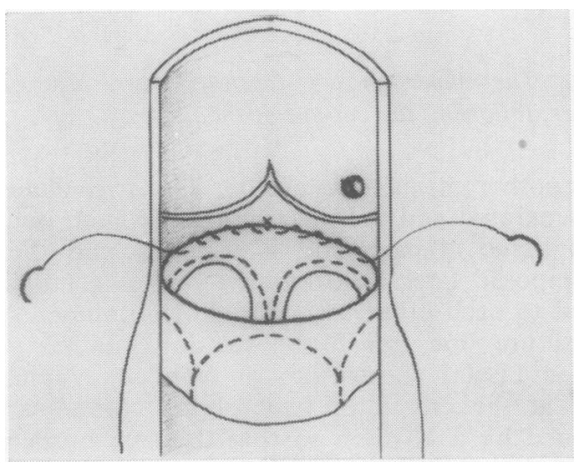

FIG. 6. The valve, turned outside in, is introduced into the left ventricle and its double ring is sutured with a running stitch just a few millimetres below the natural aortic valves.

top of the commissures, where the sutures are exteriorized by transparietal stitches. The corresponding heads of sutures are then tied on fragments of fascia outside the aorta (Fig. 7). We then close the aortotomy, taking care not to deform the valve at this stage of the procedure.

\section{CLINICAL APPLICATION}

The first aortic valve replacement as described was performed on a 33-year-old man, who presented signs of an active bacterial endocarditis. This patient had been in bed since November 1969 suffering from influenza associated with arthralgies which had been treated symptomatically. Back to work for a few days in December, the patient was extremely fatigued. Signs of sepsis were noted. A murmur of aortic insuffi- ciency was then discovered but was dismissed as un- $\vec{\theta}$ important. The patient was treated at home with.antibiotics without any improvement. He was admitted $\square$ to hospital in February 1970 and blood cultures? revealed the presence of Streptococcus viridans. Repeated antibiotic treatment was inefficient, in spite of very high doses of penicillin $(50,000,000$ units per응 day) and streptomycin ( $2 \mathrm{~g}$ per day); deterioration $\varrho$ was rapid and on March 19 the patient was transferred $\overrightarrow{\hat{\sigma}}$ to the cardiac surgery department.

On admission he was febrile, showing signs of $\vec{\supset}$ cardiac insufficiency with a blood pressure of $160 / 0=$ $\mathrm{mmHg}$, and an erythrocyte sedimentation rate of

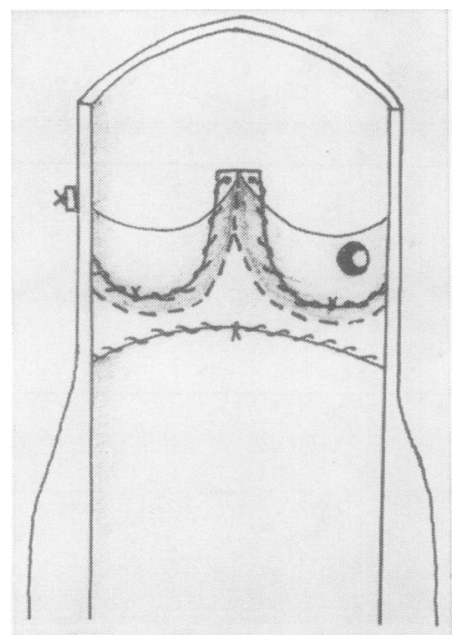

FIG. 7. The valve evaginated into the aorta is sutured in a running fashion to the corresponding insertion of the cusps on the aorta. Sutures at the commissures ares exteriorized and tied over a fascial sheet. 


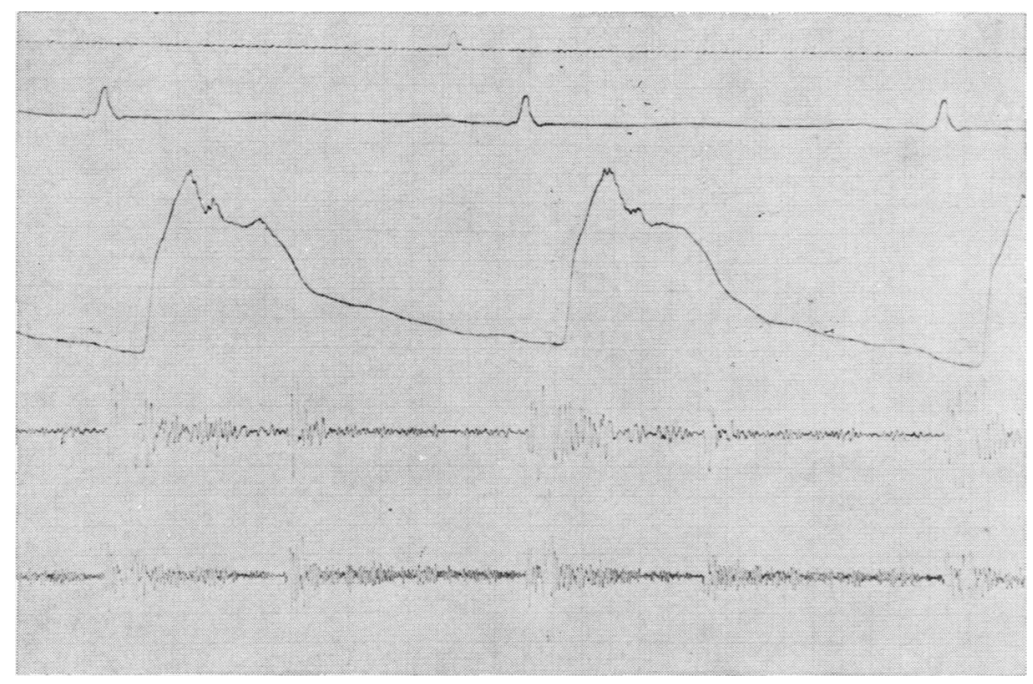

FIG. 8. Preoperative phonocardiogram showing an intense ejection click, an ejection murmur, and a regurgitant diastolic murmur. Abolition of the dicrotic wave on the carotid pulse record.

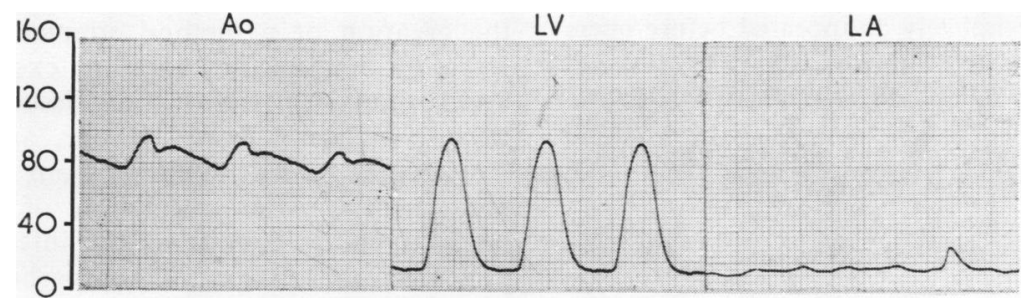

FIG. 9. Withdrawal pressure curve (aorta $\rightarrow$ left ventricle $\rightarrow$ left atrium) showing no gradient between ventricle and aorta.

$92 \mathrm{~mm} /$ hour (Westergren). There were no abnormalities on the ECG and a chest radiograph showed a prominent left lower arc. A phonocardiogram was diagnostic of a very severe aortic insufficiency (Fig. 8). The day after admission the patient was operated on. Under extracorporeal circulation and left coronary perfusion a bicuspid aortic valve- by fusion of the coronary valves-was found.

There was a marked degree of aortic insufficiency with vegetations at the base of the non-coronary valve. These vegetations extended towards the great leaflet of the mitral valve, which also showed minor ulcerations. After aortic valve excision the ring was calibrated and corresponded to a No. A 12 Starr aortic valve. A fascia lata valve was then fashioned, using the procedure already described. During this time the small lesion on the mitral valve was curetted and covered with a patch of fascia lata. The new aortic valve was then inserted. At the end of the procedure the ventilatory and haemodynamic con- ditions were perfect, and the patient was extubated on the table. Recovery was uneventful. A microcatheter introduced transeptally into the left heart during operation permitted, 24 hours later, a record of withdrawal pressure curves (Fig. 9). Antibiotics were administered for four days and the temperature became normal on the seventh day. When the patient left hospital 22 days after the operation he was in a satisfactory state. There were no signs of cardiac insufficiency or of valvular incompetence. The temperature was normal, the blood pressure $140 / 80 \mathrm{~mm}$ $\mathrm{Hg}$, and the erythrocyte sedimentation rate fell to $33 \mathrm{~mm} /$ hour. Auscultation was normal and the ECG unchanged. No anticoagulants were given. Culture of the valves as well as three blood cultures performed during the postoperative period were negative. The patient was re-examined three months later and by then he had taken up some light activity and had no significant cardiac signs other than a light protodiastolic murmur. The carotid pulse, of which the di- 


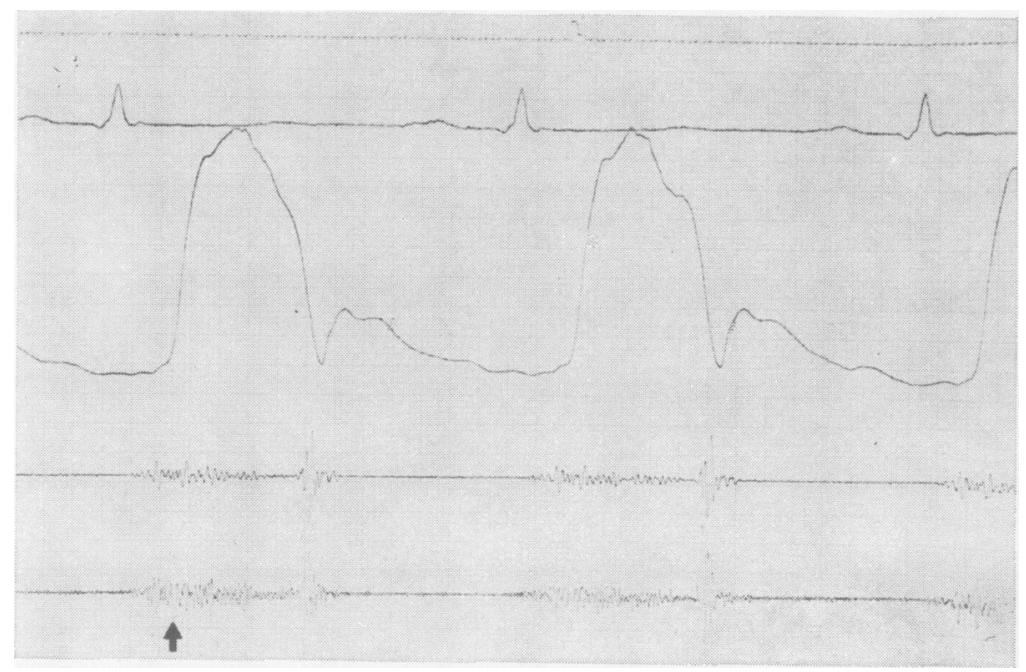

FIG. 10. Postoperative phonocardiogram showing opening snap of valve (arrowed) and following small ejection murmur. Carotid pulse shows reappearance of $a$ dicrotic wave.

crotic wave had completely disappeared before operation, had returned to normal (Fig. 10). The erythrocyte sedimentation rate was $10 \mathrm{~mm} /$ hour. Blood pressure was $140 / 80 \mathrm{mmHg}$ with a heart rate of $80 /$ minute. The ECG tracing was normal and the chest radiograph showed a normal cardiac profile.

\section{DISCUSSION}

Ionescu and his associates (1970) have extensively reviewed the literature concerning autologous fascia lata valvular replacement. Fascia lata mounted on a prosthetic support has the advantage that the valve is constructed before the aorta is opened. However, the prosthetic support used (Ionescu et al., 1970 ; Edwards et al., 1969) represents in itself a rigid obstacle to ventricular ejection. A ventriculo-aortic gradient is thus created. Furthermore, the advantage of autologous tissue should not be tempered by the simultaneous introduction of foreign material, especially in a case with infection. Valvular fascia lata replacement, using Senning's technique, is long and tedious. Barratt-Boyes' elegant technique of inserting aortic homografts has stimulated us to create a fascia lata valve shaped so as to permit an insertion similar to his. This necessitated the creation of a method adapted to rapid con-응 struction of the valve, avoiding excessive handling $ᄋ$ and trauma of the fascial tissue, such as we describe (Barratt-Boyes, Roche, and Cole, 1969). $\overrightarrow{\overrightarrow{0}}$

Our first patient suffered from acute bacterial 3 endocarditis. After correction of the abnormal haemodynamic conditions the infection soon disappeared, this in spite of, or perhaps because of, the early suppression of all antibiotic treatment.

\section{REFERENCES}

Barratt-Boyes, B. G., Roche, A. H. G., and Cole, D. S.O (1969). Aortic homograft valve replacement: a reviewß of 511 patients with a late follow-up extending to 650 months. J. cardiovasc. Surg. (Torino). Special Issuedevoted to the XVIIth Congress of the European Society $D$ for Cardiovascular Surgery, London, 1968, p. 18.

Edwards, W. S., Karp, R. B., Robillard, D., and Kerr, A. R. (1969). Mitral and aortic valve replacement with fasciaos lata on a frame. J. thorac. cardiovasc. Surg., 58, 854. N

Ionescu, M. I., Ross, D. N., Deac, R., Grimshaw, V. A., Taylor, S. H., Whitaker, W., and Wooler, G. H.W (1970). Autologous fascia lata for heart valve replace-g ment. Thorax, 25, 46.

Reid, K. (1970). The anatomy of the sinus of Valsalva. Thorax, 25, 79.

Senning, Å. (1967). Fascia lata replacement of aortic valves.' $J$. thorac. cardiovasc. Surg., 54, 465. 